\title{
Quanta and Space
}

\section{Mduli Y*}

Cape Town, South Africa

*Corresponding author: Mduli Y, Cape Town, South Africa, Email:

mduliyanga@gmail.com

\section{Research Article \\ Volume 2 Issue 1}

Received Date: January 31, 2019

Published Date: May 14, 2019

DOI: $10.23880 /$ ijnmrs-16000111

\section{Abstract}

The current fundamental theories of physics are quantum field theories (i.e. QFTs) in the form of the standard model and general relativity (i.e. GR). All current researches are completely devoted to finding a quantum theory of gravity. What we ask and try to answer is, isn't possible that quantum theory as a framework is just not the foundation of fundamental physics and neither is GR. And our answer being an alternative framework which regards matter and light as composed of indivisible and immutable units e.g. photon, electron, neutrino, up quark, masons, baryons, nuclei, atoms and molecules. Space has intrinsic energy just as photons and electrons do, its energy is finite just as theirs and this energy is directly proportional to the volume of space. And change is the annihilation of quanta or of space with all quanta and creation of new quanta or of new space with new quanta. As a quantum is immutable and so is space then our theory of gravity will be a special relativistic theory of gravity. Since space has energy and energy is conserved and the energy of space plus that of all the quanta in the universe is conserved thus space can't increase in size indefinitely but must reach maximum volume as the energy of the universe is finite, this true even for an accelerated expansion. This framework provides a new potential foundation of physic not based on the concept of force and acceleration or field theory. This paper is an elaboration of my earlier paper titled The Quanta, published in the journal of nuclear and particle physics at www.sapub.org in august 2018.

Keywords: quantum field theories; quantum theory; energy level; Daltonian model

\section{Introduction}

We ask the reader to go with us through the journey of how physicists come to have quantum theory. The experimental facts and theories which led them ultimately to this theory. Absorption and emission spectral lines, the discovery of the electron, the photoelectric effect and black body radiation and other such experimental facts forced upon physicists to abandon slowly and with resistance some of the core ideas of classical physics. Now we hope to show that maybe an alternative theory can do better than quantum theory and that possibly the idea of harmonic oscillators even quantum ones is not the right path of the comprehension of these experiments which we regard as the very foundation of our beautiful quantum theory. The theoretical system we present below is our attempt to show how this new theory could possibly account for such foundational experiments.

A quantum is an indivisible unites of light and matter e.g. a photon, electron, neutrino, mason, baryon, nuclide, atom and molecule. A quantum though indivisible it's not indestructible, a quantum can be annihilated and created. In the model to be presented here as postulated above masons, baryons, nuclides, atoms and molecules are indivisible unites of matter but unlike Dalton's 
indivisibles ours are not indestructible as stated above. Another difference from Daltonian indivisibles is that our indivisibles have electric properties and other types of charges such as color charge, in this aspect our indivisibles are more akin to the model of J.J Thomson though unlike it they are not composite systems. If molecules are indivisibles and hydrogen-chloride is made up of hydrogen-chloride molecules thus a hydrogenchloride molecule is indivisible, hence the concept of bonds is rendered unnecessary, yet chemistry teaches the experimental fact that hydrogen-chloride can be reduced to hydrogen and chlorine via a photochemical reaction among other possible means of reduction. This fact can be easily account for in our model, as our indivisibles are not indestructible. The indivisible hydrogen-chloride molecule is annihilated together with the photon when it collides with the molecule and two atoms of different species are created, one hydrogen atom and one chlorine atom. The atoms are also indivisibles. JJ Thomson's work and chemicals facts show that atomic hydrogen can be reduce to electrons and protons.

In our model these facts don't threaten our postulate that the hydrogen atom is an indivisible unite of atomic hydrogen. As the hydrogen atom is simply annihilated in such reactions and the electron and proton created. Such an explanation would have been impossible in a Daltonian model as it regards atoms of elements as not only indivisible but indestructible, our model does away with the idea of indestructibility and is successful where the Daltonian model failed. Our models achieve this by doing away with the idea of indestructibility. We hope the reader sees how a model which regards molecules and atoms as indivisible can possibly be compatible with experiments and observations which at first sight seem to contradict this model. of course, the Daltonian model is not compatible with such experiments and observation as these facts contradict the model of J Dalton.

E Rutherford's work demonstrated that JJ Thomson's model was an inadequate representation of the atom as it led to conclusions which contradicted experiments. Based on the celebrated gold-foil experiment Rutherford concluded in light of major deflection of alpha particles that the positive charge of the atom must occupy a very small volume and must carry almost all the mass of the atom. In Rutherford's model the positive charge in an atom resides in a very small volume in comparisons to the dimensions of atom and is orbited by electrons.

Now in our model an atom is an indivisible unite and thus is not made of electrons and nucleus as in Rutherford's case or in the Thomson case electrons imbedded in mass of positive charge, thus the volume of the atom has nothing to do with the volume of the electron or nucleus. Our model provides a different interpretation of the gold-foil experiment where alpha particles collide with gold atoms, each alpha particle collides with one gold atom both being annihilated and an ion of gold, electrons and alpha particle created such that other alpha particles approach these ions of gold and are deflected. Thomson's models could not possibly explain the experimental findings as it regards the volume of the atom being basically that of the positive mass hence leading to the inability of explaining the fact that the positive charge must have a small volume inorder to deflect the energetic alpha particles. So, the volume of the gold nuclei differs from that of a gold atom and the volume of the gold atom is not the result of how far electrons orbit about the nucleus as there no nuclei or electrons in the atom as it's an indivisible entity. In our model the atom is indivisible and this indivisibility means that the atom is stable unlike the Rutherford model which leads us to believe that atoms should be unstable systems that quickly collapse.

$\mathrm{N}$ Bohr's work is the modification of Rutherford's model and applies Plank's quantum hypothesis in order to explain the discrete nature of absorption and emission spectra of atoms and their being unique for each element. Herein is born the idea that the electron can only occupy certain energy-levels and also the idea of quantum jumps. Where absorption consisted in an electron jumping to a high energy-level via absorption of electromagnetic radiation and emission being a jump from higher energylevel to a lower one or directly to the ground state.

The reader now may ask himself how are these spectra to be made explicable in a model which regards an atom as indivisible unite even with the idea that it can be created and annihilated. The answer isn't as hard and unnatural as might be expected rather what featured in Bohr's models as an excited atom where an electron is in a high energy-level, in our model the excited atom is an indivisible unite which is unstable as it has a rest mass that is higher than its unexcited counter-part. Thus absorption is the annihilation of a photon and an atom with creation of an excited atom. And since the excited atom is unstable, it decays or is annihilated and a photon plus an unexcited atom are created. Our model is capable of explaining discrete spectra without a composite model of the atom, quantum-states or jumps. In Bohr's model and all quantum mechanical models of the atom since then to the current models of the 21st century an electron has a choice when it is in a high energy-level to leap directly to the ground-state or go to an intermediate state. But our model clearly states that an excited atom can only decay to an uncited atom plus a photon. As any unstable 
particle doesn't just decay to any set of products with a lower sum rest mass than its own but to the set of products with the least sum rest mass. This principle just stated now helps us escape the probability of transitions which is ever present in any quantum model of the atom. According to this model the energy of the photon that is created during emission is given by this equation $\mathrm{E}(\gamma)=$ $\left(\mathrm{m} 0-\mathrm{m} 0^{\prime}\right) \mathrm{c} 2, \mathrm{~m} 0$ is the rest mass of an excited atom and $\mathrm{m} 0^{\prime}$ is that of the unexcited atom this equation differs from that of Bohr in that it has nothing to do with orbital radii. If the excited atom is at rest i.e. its momentum is zero then it will decay to two identical photons with opposite momenta with this equation $2 \mathrm{E}(\gamma)=\mathrm{E}(\gamma)=(\mathrm{m} 0$ $\mathrm{m} 0$ ')c2. Thus we do away with the idea of energy levels and quantum leaps and the rift it causes between special relativistic mechanics and our models of atoms regards the motion of particles in a potential gradient. Now we present our model of the hydrogen atom (i.e. protium) there exists the unexcited hydrogen atom which has the least rest energy of all and excited hydrogen atoms which have higher rest energy than that of the unexcited one. All these atoms are indivisible, all the excited atoms decay to the unexcited one plus photons this explains discrete line spectra. Thus the rest energy of any excited atom of hydrogen (protium) is greater than the rest energy of the unexcited atom and lesser than the sum rest energy of a proton and electron. Therefore, we can write E0(1H0) the rest energy of an unexcited hydrogen atom, E0(1H10*) the rest energy of the first excited atom, E0 $\left(1 \mathrm{H} 20^{*}\right)$ and so on. So we can write $\mathrm{E} 0(1 \mathrm{H} 0)<\mathrm{E} 0\left(1 \mathrm{H} 10^{*}\right)<$ $\mathrm{E} 0\left(1 \mathrm{H} 20^{*}\right) \ldots$ and can go on to explore the possible decay reactions of the unstable hydrogen atoms to stable hydrogen atom and photons. Here we begin with the simplest case that of an excited atom with zero kinetic energy, here are the reactions $\left(1 \mathrm{H} 10^{*}, 1 \mathrm{H} 0\right) 2 \gamma$ and $\left(1 \mathrm{H} 20^{*}, 1 \mathrm{H} 0\right) 2 \gamma$ and so on for excited atoms of higher rest energy. Now for reasons of momentum conservation when an excited atom has nonzero momentum i.e. nonzero kinetic energy it can't decay two identical photons and an unexcited atom, and each excited atom when its kinetic is greater than the ionisation of the unexcited atom minus the difference in rest energy of the excited atom and the unexcited atom then the excited atom decays to a proton and electron. If an excited hydrogen atom's energy given by its rest energy plus kinetic energy is greater the sum rest energy of a neutron, a neutrino and $w$-particle it decays to these particles. Now for the helium spectral lines the same is true, we can discuss helium-4 atoms as this is as the most abundant isotope of helium. One can ionise a helium-4 atom to an electron and an ion. The helium ion can be further ionised to a helium nuclide and an electron. All that has be described can be done via ionisation of the helium atom to a nuclide and two electrons. Now if we apply the same general ideas we used in constructing a model of the hydrogen atom to the case of helium atoms we arrive a result alien to quantum mechanical models of the atom that is a sample of helium atoms will only emit spectral lines unique to unionised helium if the atoms are not ionised and only emit spectral lines unique ionised helium ions when all atoms are ionised or a mixture of both spectra if the sample is composed of both atoms and ions. And according to this scheme if the ionisation of an ion of an element is lesser than that of the atom even if a photon has enough energy to ionise the ion and not enough to ionise the atom the won't be any ionisation contrary to the quantum model which allows for such a photon to ionise the atom. We can discuss the spectra of radioactive atoms; the tritium atom will serve as our exemplar of radioactive species. Now an uncited tritium atom with zero kinetic energy decays to a helium-3 atom, a neutrino and a w-particle. And all excited tritium atoms with zero kinetic energy also decay to a helium-3 atom, a neutrino and a w-particle. The result of this is that all substances made up of radioactive atoms have no atomic emission spectra, thus they have only absorption spectra. Now a tritium atom with kinetic energy greater than its ionisation energy decays to a tritium nuclide and an electron. This is supported by experiments in as far as we are aware for tritium has no emission spectra as far as experiments have probed.

Heisenberg's matrix mechanics with its uncertainty principle and Schrödinger's wave mechanics, in these physicists' work the idea of a quantum harmonic oscillator is developed further than it was in the case of Plank, the oscillator is treated as a particle trapped in a potential gradient and energy levels explained via the idea of standing waves hence providing a foundation for Bohr's stationary states or orbits. The basic foundational equation of wave mechanics describes the total energy of a system as being given by or equal to the potential energy plus kinetic energy of the system. E = Ep + Ek. In this theory an electron can only be released from the atom if a photon has energy equal to or greater than the ionisation energy of the atom. In the hydrogen case this energy is 13.6 or something close to that figure now a photon's energy must be 13.6 or greater to ionise hydrogen. $\mathrm{E}(\gamma)=\varphi, \mathrm{E}(\gamma)$ is the energy of the photon and $\varphi$ is the ionisation energy.

M. Born explained that the square of the wave function is the probability of finding an electron at particular region about the nucleus of the atom. And there are four quantum numbers explaining the behaviour of the electron in an atom. The famous Pauli's exclusion principle is the fourth of these quantum numbers called 


\section{International Journal of Nuclear Medicine \& Radioactive Substances}

the spin quantum number, Bohr's one is the principal quantum.

Since in our model the hydrogen atom is indivisible there's zero probability of finding where an electron is in the atom as there's no electron or proton for that matter and also there can be no quantum tunnelling. And the need which was necessary at the time to describe the electron as a waveform in order to reconcile the Bohr model with experiments which led to the uncertainty principle proves superfluous in our model as the atom is indivisible. The idea of a quantum oscillator which can only have discrete energy levels thus accounting for spectral lines is made unnecessary. And finally the energy of an atom unlike in the quantum mechanical case is given by the rest energy of the atom plus kinetic energy as the atom is not a composite system thus the idea of potential energy never arises. $E=E 0+E k=\gamma m 0 c 2$. In our model what is necessary is only this that the energy of the atom plus that of the photon be greater the sum rest energy of the electron plus that of the proton which implies that all that is necessary is for the kinetic energy of the atom plus the energy of the photon to be greater than the ionisation energy. $\operatorname{Ek}(A)+E(\gamma)>\varphi, E k(A)$ is the kinetic energy of the atom and $\varphi$ is the ionisation energy. Here we see that the energy of the photon need not be equal to the ionisation energy unless the atom has zero kinetic energy if it has non-zero kinetic energy then a photon with energy lesser than the ionisation energy can ionise an atom. Thus we see that our model makes predictions which differ from those of the quantum model infact the two models contradict each other, and herein lies the hope of experimentally checking which one captures our experimental facts more faithfully. Thus according to our model it is easier to ionise an atom with high kinetic energy that it is with one of low kinetic energy i.e. atoms with high kinetic energy require lesser energy to be ionised than do atoms of lesser kinetic energy. Our model interprets ionisation as the annihilation of an atom and the creation of an ion plus electron(s).

Dirac's quantum electrodynamics, which explained the creation and annihilation of photons by viewing photons as quantum excitations of the electromagnetic field. Here photons can only be created by the excitation of the photon field by charged matter fields [1].

Ours is not a field theory and hence not a quantum field theory either and there are no quantum states. The reason for the lack of quantum states is the indivisibility of molecules, atoms, nuclides and such which leads to a different idea as to what emission is and that it has nothing to do with the oscillation of electric charges.
J Chadwick discovers the neutron and the theory of the nucleus is modified in light of this discovery. Heisenberg develops a nuclear model and Pauli postulates the neutrino to save the law of conservation in beta decay.

Fermi develops his theory of beta decay by regarding matter particles as excitations of matter fields and that the proton, electron and neutrino are created during beta decay.

Our theory offers a different theory of the nucleus as it regards all nuclides as indivisibles thus nuclides are not made up of protons and neutrons so the need for a strong force doesn't arise. Of course in our model one can annihilate a nuclide and create nucleons. And its interpretation of beta decay differs from that offered by Fermi's theory. For every unstable nuclide there exists one stable nuclide which is its isobar. The result of this idea is that all heavy unstable nuclei such as uranium nuclei, plutonium, neptunium and other heavy nuclides decay to stable isobars when they have low kinetic energies such as near zero or zero kinetic energy. Thus if this idea is true then a uranium nuclide with zero kinetic energy must decay to a stable nuclide. A uranium nuclide can only decay to an alpha particle plus another nuclide if the energy of the uranium nuclide is greater than the sum rest energy of the created product particles as the sum rest energy of the alpha plus that of the product nuclide is greater than the rest energy of uranium nuclide.

$\begin{array}{lll}{ }^{2} \mathrm{H} & & \\ { }^{3} \mathrm{He} & { }^{3} \mathrm{H} & \left({ }^{3} \mathrm{H}^{0},{ }^{3} \mathrm{He}^{0} v_{\mathrm{e}}\right) \mathrm{w} \\ { }^{4} \mathrm{He} & & \\ & \\ { }^{6} \mathrm{Li} & & \\ { }^{7} \mathrm{Li} & { }^{7} \mathrm{Be} & \left({ }^{7} \mathrm{Be}^{0},{ }^{7} \mathrm{Li}^{0} v e\right) \mathrm{w} \\ & & \\ { }^{9} \mathrm{Be} & { }^{9} \mathrm{~B} & \left({ }^{9} \mathrm{~B}^{0},{ }^{9} \mathrm{Be}^{0} v_{\mathrm{e}}\right) \mathrm{w} \\ { }^{10} \mathrm{~B} & { }^{10} \mathrm{Be} & \left({ }^{10} \mathrm{Be}^{0},{ }^{10} \mathrm{~B}^{0} v_{\mathrm{e}}\right) \mathrm{w} \\ { }^{11} \mathrm{~B} & & \\ { }^{12} \mathrm{C} & & \\ { }^{13} \mathrm{C} & & \\ { }^{14} \mathrm{~N} & { }^{14} \mathrm{C} & \left({ }^{14} \mathrm{C}^{0},{ }^{14} \mathrm{~N}^{0} v_{\mathrm{e}}\right) \mathrm{w} \\ { }^{15} \mathrm{O} & & \\ & \end{array}$

The rows are periods and the columns are groups. There are five groups in total as there exists from experiment only nuclides which can undergo single or double beta decay. Only the elements in group one are stable and all other groups have unstable elements. All the elements in a period except for the first element decay to the first element when their kinetic energy is close to zero or is zero. There are empty periods such as period four 
and period 7 and so on because there are no nuclei with such numbers of nucleons. An element with isotopes that all occupy group one has only stable isotopes e.g. helium. An element with isotopes that all occupy groups greater than one has only unstable isotopes e.g. uranium. Hydrogen is an example of an element with both stable and unstable isotopes.

Period one has one element, two has two elements, three has one, four has zero, five has one, six has two elements, seven has zero, eight has two and so on. The Lambshift and Feynman's QED with virtual particles, with its use of renormalisation in order to evade the infinities which plagued QED in those days.

The Lambshift is compatible with our model of the hydrogen atom and there's no need for virtual particles because the hydrogen atom is according to the model presented here not a composite system. The quark model, the electroweak theory and quantum chromodynamics. In our model nucleons are indivisible and can be destroyed creating quarks as each nucleon has the properties of three quarks but no quarks inside it bound by virtual gluons and quark antiquark pairs. Thus there's no need for the color force if this model be true. Here we present the axioms upon which our model is based. The first of these has already been stated at the beginning of this paper. A quantum is an indivisible unite of matter and light e.g. photons, leptons, hadrons, nuclides, atoms, molecules and excited hadrons, excited nuclides, excited atoms and excited molecules. Thus there are no electrons which emit photons inside atom or molecules and there are no bonds, valence electrons and exclusions principles and orders of filling orbitals and quantum leaps, energy levels all other concepts which the quantum mechanical model of the atom and molecules so intractable and complicated. Conservation of quanta which is not conservation in number but in that all quanta arise from the annihilation of other quanta, thus no quanta can arise where there was no quanta even if all conservation are not violated. And when created quanta are annihilated other quanta are created, thus quantum can't be annihilated without the creation of other quanta. All massless quanta are photons. Consequence no other kind of massless particles exist e.g. gluons and gravitons.

All massive quanta are matter quanta. Consequence the $\mathrm{Z}, \mathrm{W}$ and $\mathrm{H}$ (higgs) bosons are matter particle and the $\mathrm{ZO}$ and $\mathrm{HO}$ bosons are particle of the same kind as positronium and neutral-pi-mason and such other particle which are Majorana matter particle. All quanta obey the energy-momentum relation of special relativity [2]. Consequence there exists only on-shell-mass particle thus no virtual particles. All quanta have nonzero volume therefore they are not microblack holes. Each quantum exists for a nonzero duration i.e. $\Delta \mathrm{t}^{\prime}>0, \Delta \mathrm{t}^{\prime}=\gamma \Delta \mathrm{t}$. Conservation of Dirac properties e.g. conservation of electric charge or color charge. Decay reaction is the annihilation of one unstable quantum and the creation of two or more quanta, where the number of created quanta is greater or equal to two but less than infinity i.e. $2 \leq \mathrm{n}<$ $\infty$.

Collision reaction is any contact reaction between two or more quanta and the creation of one or more quanta i.e. $1 \leq n<\infty$. During a reaction where the reactants have energy only for products with a sum rest mass lesser than their own they have only those products with least sum rest mas and when they have energy to have products with sum rest mass greater than their own they have only those products with the greatest sum rest mass greater than their own. The consequence of this is that a positronium or neutral-pi-mason or particle of the same kind such as $\mathrm{ZO}$ or $\mathrm{HO}$ boson with zero momentum can only decay to photons and not to particles of sum rest lesser than their own which is nonzero as photon have zero rest and sum of them has zero rest mass thus they have the least possible sum rest mass and thus the only matter product reaction they can are products with sum rest energy greater than their own e.g. $(\mathrm{p} 0,2 \gamma)$ is possible while it decaying to a neutrino and an anti-neutrino is impossible but (p0,e-)e+ is possible. And if tow photons have energy greater than the sum rest mass of two charge pi-masons their products will be the $\pi-+\pi+$ and not an electron and positron or a muon and an anti-muon as the rules says the products must be those with the greatest sum rest mass greater than that of the reactants. Thus the products of a quantum that decays at rest are all stable quanta. Another result of the principle is that two photons will create a particle and an anti-particle if their energy is greater than that of the pair of matter particles and since two visible light photon don't create any of the neutrinos it follows that the electron and anti-electron neutrino has a sum rest energy greater that 2(3.1)eV. The other consequence is that the time-reversal symmetry is broken to a higher degree that is the case in the standard model, as an electron and positron can't create positron plus photons though positron with enough energy does create an electron plus positron. And though an electron and positron can create a muon plus anti-muon when their energy is greater than that of the sum rest energy of the muon and anti-muon, the muon anti-muon pair can't create the electron positron pair as it creates photons then when it has enough energy a neutral pion and if they have even higher energies they can create the charged pion pair. This reaction is irreversible $\mathrm{e}+(\mathrm{e}-, \mu+) \mu$ - and this one is reversible $\mathrm{e}+(\mathrm{e}-, 2 \gamma)$ and this one is irreversible $(\mathrm{p} 0, \mathrm{e}+) \mathrm{e}-$ and also this one is irreversible $\mathrm{p}(\gamma, \mathrm{e}+\mathrm{e}-\mathrm{e}$. 
The rule of mins i.e. during any reaction where the reactants have products with the least sum rest mass or the greatest sum rest mass the number of products quantum must be the minimum possible such that no laws are violated and when the reactants have products with the same sum rest mass as theirs own the not only is the number of particles conserved in such a reaction but also the number of types of particles is conserved. Any even number of photon can create a stationary positronium but positronium with zero momentum decays only to two photon this is another form of the breaking of time-reversal symmetry because it means that even if positron is created by four identical photons with zero sum momentum the positronium particle will decay to two photons making the reaction with four reactant photons irreversible.

An unstable quantum is any quantum which when at rest there exists a set of quanta with a sum rest mass lesser than its own it can decay to without violating any laws. Thus photons are stable and all unstable quanta are matter quanta. Any unstable quantum has these intrinsic properties volume, energy (rest and kinetic energy), Dirac properties, spin, linear momentum and life-span. Any Two quanta with identical volume, energy, Dirac properties, spin and linear momentum have the same life-span. A stable quantum is any quantum which when at rest there exists no quanta with a sum rest mass lesser than its own it can decay to without violating any laws. Any stable matter quantum has all the intrinsic properties of an unstable quantum with the exception of life-span.

Now it's clear that according to the rules stated above the hydrogen atom as it is stable i.e. the unexcited hydrogen atom can't decay to any particle no matter how much kinetic energy it has. On the other hand an excited hydrogen will have different decays depending on its kinetic energy e.g. When the excited hydrogen atom is at rest $\left(1 \mathrm{H} 0^{*}, 1 \mathrm{H} 0\right) 2 \gamma$, when moving possibly $(1 \mathrm{H} 0 *, 1 \mathrm{H} 0) \gamma$ and when its energy is greater than the sum rest mass of the electron and proton i.e. $\mathrm{E}\left(1 \mathrm{H} 0^{*}\right)=\mathrm{E} 0\left(1 \mathrm{H} 0^{*}\right)+$ $\mathrm{Ek}\left(1 \mathrm{H} 0^{*}\right)>\mathrm{E} 0(\mathrm{p}+)+\mathrm{E} 0(\mathrm{e}-)$ then $\left(1 \mathrm{H} 0^{*}, \mathrm{p}+\right) \mathrm{e}-$. This is impossible in any classical system and also in quantum theory with that exact condition as stated above without probabilities [3].

There are four Dirac properties the color charge, the electric charge, the neutrino charge and the w-charge. Quarks have all four charges, the charge leptons have the three charges the electric charge, neutrino charge and wcharge. Neutrinos have the two charges the neutrino charge and w-charge. The $\mathrm{w}$ particle has one charge the $\mathrm{w}$-charge. Now each fundamental particles can only have one elementary charge e.g. a quarks can only have an elementary color charge (i.e. either 1 or -1 ) and all its other charges are fractional all quarks have one elementary color charge(i.e. 1). The same is true of charged leptons, all charge leptons have the elementary electric charge (i.e. -1). This means that quarks just like charged leptons can't change their charges even the color charge as is the case in quantum chromodynamics. Since all hadrons are indivisible and all massless quanta are photons (i.e. real photons) the color fields are unnecessary in this framework. Elementary particles of the same kind e.g. quarks have the same elementary color charge but different fractional charges the same is true of charged leptons they have the same electric charge and different fractional charges [4].

So if it's true as experiment suggest in particles accelerators that the protons has the Dirac properties of three quarks the stability of the proton is the result of the fact that it's the smallest baryon with three color charges as the neutron also has three color charges but is not the smallest baryon. And masons are all unstable because they have no resultant color charge as they have the Dirac properties of a quark and an anti-quark.

\section{Mechanics}

This framework if true implies that there's no weak or strong force as particle decay only to stable particles when at rest or near rest energy and all masons, baryons and nuclei are indivisible. Hence the mechanics of this framework will deal with gravity and electromagnetic forces. But before that it's necessary to clarify that light in all it manifestations as visible light, ultraviolet, inferred and X-rays and gamma rays and such is not electromagnetic according to our framework as atoms don't emit because of electrons making jumps or the result of any oscillating charges. So by electromagnetic force we mean just the electric and magnet force no optical phenomenon. In this framework there are no virtual particles as all particles which exists obey the energy-momentum relation thus only on shell mass quanta exist.

Our mechanics begins with the two postulates of special relativity and adds a third one. This new postulate is a consequence of the earlier postulate that a quantum is an immutable entity. Thus, our third postulate is there existing only inertial systems (i.e. systems with constant velocities). Thus, it is clear force and acceleration are inadmissible concepts in the theoretical framework. Since space is immutable and finite in energy and volume that the leads us to our fourth axiom, space is a threedimensional finite non-Euclidean structure with a 
geometrical structure that mirrors the distribution of light and matter quanta at the moment of creation of this space and all its quanta. Thus, the geometry of the spatial component of the Minkowski spacetime of special relativity is changed from being a three-dimensional Euclidean structure to a no-Euclidean structure. This new finite non-Euclidean space exists like quanta for a nonzero duration given by the time it would take light to traverse the shortest distance between any two quanta at the momentum of creation of the space and all quanta i.e. $\mathrm{t}=\mathrm{d} / \mathrm{c}, \mathrm{d}$ is the shortest distance and $\mathrm{c}$ is the speed of light and $t$ is the time. Now we can consider the simplest case of accounting for gravity in this framework that of two quanta created with space both at rest that is with zero momentum after the time has passed which it would take light to traverse the distance between them space and both quanta are annihilated and a new space is created with two new quanta. The new quanta are of the same type as the annihilated one but are moving that is have more energy than the previous ones and space has lesser energy and thus lesser volume than the annihilated space. The energy density of an annihilated space is equal to that of the created space always. We can now discuss the case of free fall in our theoretical system given two quanta of different rest energy created from the same distance from the ground will exist for a duration given by $t=d / c$ after which both will be annihilated together with all other bodies and space with the creation of new counterparts of the annihilated quanta which have equal velocities and since they have different rest energies therefore they have different kinetic energies these new quanta will also exist for non-zero duration and be annihilated creating two new counterparts with equal velocities. Thus unlike in Newtonian mechanics or in Einsteinian mechanics where a point particle in free fall gains kinetic energy in a continuous fashion that is not the case in this theoretical scheme, since the point with zero kinetic energy is annihilated and one with kinetic energy is created which exists for a no-zero duration moving in constant velocity thus would have the same kinetic energy in different positions on its descent which contradicts both Newtonian and Einsteinian mechanic as both demand that the point particle have different kinetic energy at different positions in its descent. It's clear hopefully to the reader how our theoretical system can account for the experimental fact of the equality of inertial and gravitational mass. Since space is non-Euclidean in this framework and its geometrical structure (i.e. curvature) mirrors the distribution of light and matter at the momentum of its creation with all light and matter this accounts for gravitational lensing. If a quantum is created at the moment space is created and has the shortest distance between it and any other quantum and exists until space is annihilated then that quantum will be annihilated with space and its created counterpart in the created space will have different kinetic energy and momentum than its annihilated counterpart though still having the same rest energy as the particle type is conserved, any particle which doesn't satisfy both conditions will be annihilated with space and its created counterpart in the created space will be identical to it i.e. have the same kinetic energy, momentum and rest energy. Since space has energy and the bigger the space the more its energy in an expanding universe each annihilated space is replaced by and even bigger created space, now this can't continue forever because energy is conserved and finite thus even a universe undergoing an accelerated expansion must reach a maxim which would be the biggest created space possible and such a space would the later be annihilated a new space of lower volume created which is the beginning of a contraction epoch where the universe would be getting smaller until it reach a minimum size from which it can't get and small as space can't have zero volume as the energy density of any annihilated space must equal that of the created space.

Thus in conclusion we can say according to our theoretical system quanta which we can treat as point particles for mathematical simplicity are only capable of constant velocity motion and space is an immutable and finite simply connected non-Euclidean structure, and all gravitational and electromagnetic force effects are explained by the annihilation of space with all its quanta and the creation of new space with new quanta, thus the unification of gravity and electromagnetism is achieved as both effects are explained via the same concepts. As action at distance theories or field theories just will not do in this kind of theoretical system, since point particles are immutable i.e. can only move at constant velocities and can't change their velocities and thus can't be influenced the concept if action at distance force and by extension fields mediating influence are inadmissible and only constant velocity motion is admitted which leads to an even greater result than the unification achieved of gravity and electromagnetism i.e. the Lorentz invariance is a global symmetry in this theoretical framework even for physic where gravity can't be neglected.

\section{References}

1. Einstein A (1905) On the Electrodynamics of Moving Bodies, pp: 1-24.

2. Einstein A (1915) The General Theory of Relativity

3. Weinberg S (1995) The Quantum Theory of Fields

4. Mduli y (2018) The Quanta 8(1): (1-5). 